\title{
Theoretical Design and Circuit Implementation of Multidirectional Multi-Torus Chaotic Attractors
}

\author{
Simin Yu, Jinhu Lü, Senior Member, IEEE, and Guanrong Chen, Fellow, IEEE
}

\begin{abstract}
This paper introduces a novel fourth-order double-torus chaotic circuit. Based on this basic circuit, a systematic theoretical design approach is proposed for generating 1-D $n$ torus, 2-D $n \times m$-torus, 3-D $n \times m \times l$ torus, and 4-D $n \times m \times l \times p$ torus chaotic attractors. This is the first autonomous circuit reported in the literature for generating multidirectional multi-torus (MDMT) chaotic attractors. The dynamical behaviors of these MDMT chaotic systems are further investigated, including equilibrium points, bifurcations, Lyapunov exponents, and Poincaré maps. Theoretical analysis shows that the MDMT chaotic attractors can be generated by switching and displacing a basic linear circuit. Finally, a block circuit diagram is designed for hardware implementation of the MDMT chaotic attractors. This is also the first time in the literature to experimentally verify a maximal 1-D 20-torus, a maximal 2-D $5 \times 5$ torus, and a maximal 4-D $5 \times 5 \times 3 \times 3$ torus chaotic attractors.
\end{abstract}

Index Terms-Fourth-order double-torus chaotic circuit, multidirectional multi-torus (MDMT) attractor, saturated function series, step function series.

\section{INTRODUCTION}

A N ORDINARY torus is a special surface with a geometrical "hole." A stable torus is emerged from a super-critical Neimark-Sacker bifurcation. In general, the tori are easily observed in some two-dimensional and periodically forced dynamical systems [1]-[3]. However, it is rather rare to observe a stable torus in a 3-D or 4-D autonomous system. As a result, there are very few reports on generating tori in 3-D or 4-D autonomous systems in the literature. Moreover, physical circuit implementations of various torus attractors are quite difficult [3]-[5].

In the torus-doubling route to chaos, the original torus appears to split into two circles at the torus-doubling bifurcation point.

Manuscript received January 20, 2007. This work was supported in part by the National Natural Science Foundation of China under Grant 60221301 and Grant 60572073, by the National Basic Research Program (973) of China under Grant 2007CB310800, by the Scientific Research Startup Special Foundation on Excellent Ph.D. thesis and Presidential Award of The Chinese Academy of Sciences, by the Important Direction Project of Knowledge Innovation Program of Chinese Academy of Sciences under Grant KJCX3-SYW-S01, by the Natural Science Foundation of Guangdong Province under Grant 32469 and Grant 5001818, by the Science and Technology Program of Guangzhou City under Grant 2004J1-C0291, and by the Hong Kong Research Grants Council under the CERG Grant CityU 1114/05E.

$\mathrm{S}$. Yu is with the College of Automation, Guangdong University of Technology, Guangzhou 510090, China.

J. Lü is with the Key Laboratory of Systems and Control, Institute of Systems Science, Academy of Mathematics and Systems Science, The Chinese Academy of Sciences, Beijing 100080, China, and also with the Department of Ecology and Evolutionary Biology, Princeton University, Princeton, NJ 08544 USA (e-mail: jhlu@iss.ac.cn).

G. Chen is with the Department of Electronic Engineering, City University of Hong Kong, Hong Kong (e-mail: gchen@ee.cityu.edu.hk).

Digital Object Identifier 10.1109/TCSI.2007.904651
In fact, the so-called torus-doubling route to chaos is reminiscent of the period-doubling route to chaos. As far as we know, previous works on torus breakdown only focused on numerical simulation and experimental observation of a single torus or a double-torus [1]-[5]. Therefore, it is very interesting to ask whether there exists a simple third- or fourth-order autonomous circuit that can generate MDMT chaotic attractors. This paper gives a positive answer to this question.

To begin with, it is necessary to briefly review some main advances in multiscroll chaotic attractor generation. Over the last two decades, the theoretical design and hardware implementation of various complex multiscroll chaotic attractors have been a subject of increasing interest due to their potential applications in various chaos-based technologies and information systems [5]-[28]. Historically, Chua first discovered Chua's double-scroll circuit [1], [2]. Suykens and Vandewalle then [8], [9] proposed a family of $n$-double scroll chaotic attractors. Suykens and Chua [10] also introduced $n$-double scroll hypercubes by using 1-D cellular neural networks (CNN). Aziz-Alaoui [11] investigated multispiral attractors in both autonomous and nonautonomous systems. Tang et al. [13], [14] proposed a sine-function approach for generating $n$-scroll chaotic attractors, with a systematical circuit realization that can physically produce up to as many as ten scrolls visible on the oscilloscope. Lü et al. [15], [16] presented a switching manifold method for creating chaotic attractors with multiple merged basins of attraction. Yalcin et al. constructed a family of scroll grid chaotic attractors [17], including 1-D $n$-scroll, 2-D $n \times m$-grid scroll, and 3-D $n \times m \times l$-grid scroll chaotic attractors. Lü et al. [18]-[20], [26] introduced the hysteresis and saturated functions series approaches for generating 1-D $n$-scroll, 2-D $n \times m$-grid scroll, and 3-D $n \times m \times l$-grid scroll chaotic attractors, with rigorously mathematical proof and physical realization for the chaotic behaviors. Yu et al. [28] proposed a general jerk circuit for creating various types of $n$-scroll chaotic attractors. Recently, Lü and Chen [3] reviewed the recent advances in theories, methods, and applications of multiscroll chaos generation. In particular, the high complexity of multidirection multiscroll (or torus) attractors compared with the single scroll attractors means the generally applied prospect in the chaos-based information technologies.

In the following, a novel fourth-order circuit is constructed for generating a double-torus chaotic attractor. Based on this basic circuit, a systematic theoretical design approach is developed for creating 1-D $n$-torus, 2-D $n \times m$-torus, 3-D $n \times m \times l$-torus, 4-D $n \times m \times l \times p$-torus chaotic attractors. This is the first autonomous circuit reported in the literature for generating multidirectional multi-torus (MDMT) chaotic attractors. The dynamical behaviors, including equilibrium points, bifurcations, Lya- 


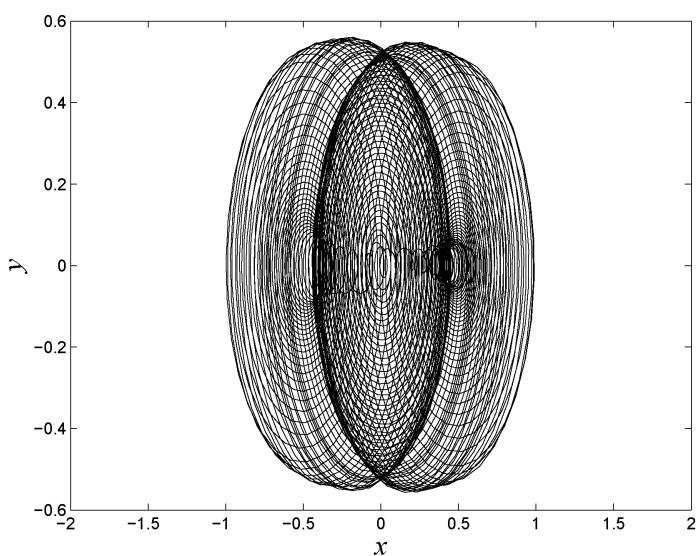

(a)

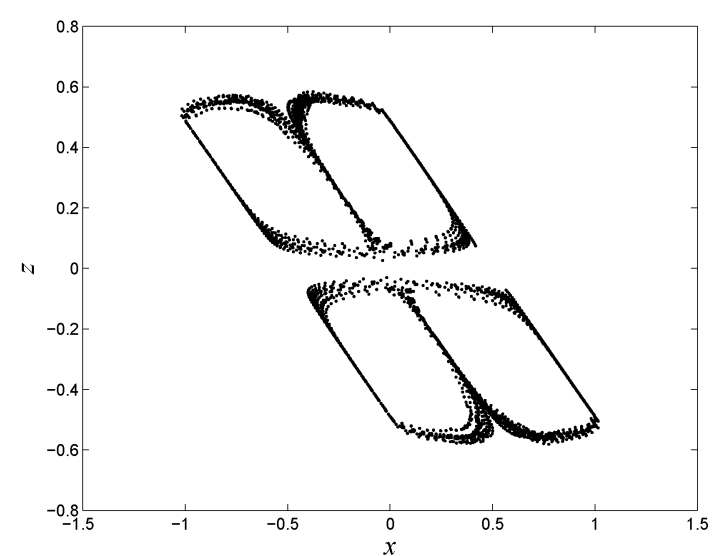

(b)

Fig. 1. Double-torus chaotic attractor. (a) $x-y$ plane projection. (b) Poincaré map on $x-z$ plane with $y=0$.

punov exponents, and Poincaré maps, are further investigated for the MDMT chaotic system. Furthermore, theoretical analysis reveals that the MDMT chaotic attractors can be generated by switching and displacing a basic linear circuit. In addition, a novel block circuit diagram is designed for physically realizing the MDMT chaotic attractors. This is the first time in the literature to physically realize a maximal 1-D 20-torus, a maximal 2 -D grid $5 \times 5$ torus, and a maximal 4 -D grid $5 \times 5 \times 3 \times 3$ torus chaotic attractors.

The rest of this paper is organized as follows. In Section II, a simple fourth-order double-torus chaotic circuit is designed and its dynamical behaviors are investigated. A systematic theoretical design approach is then proposed for generating MDMT chaotic attractors in Section III. In Section IV, a novel block circuit diagram is constructed for hardware implementation of the MDMT chaotic attractors. Conclusions are finally drawn in Section V.

\section{Simple Fourth-ORder Double-Torus CHAOTIC Circuit}

This section introduces a simple fourth-order double-torus chaotic circuit. Some typical dynamical behaviors of this system are then explored.

\section{A. A Simple Fourth-Order Double-Torus Chaotic Circuit}

We propose a novel fourth-order double-torus chaotic system, described by

$$
\left\{\begin{array}{l}
\frac{d x}{d \tau}=y \\
\frac{d y}{d \tau}=z \\
\frac{d z}{d \tau}=w \\
\frac{d w}{d \tau}=-\alpha x-\beta y-\gamma z-\delta w+\alpha f_{1}(x)
\end{array}\right.
$$

where $\alpha, \beta, \gamma, \delta$ are system parameters and $f_{1}(x)=$ $A_{1} \operatorname{sgn}(x)$ with $A_{1}>0$. In this section, assume that $\beta=\gamma=\delta=1$ and $A_{1}=0.5$. Elwakil investigated this model [6], but not for the torus chaotic attractors.

When $\alpha=0.1$, system (1) has a double-torus chaotic attractor as shown in Fig. 1(a). Fig. 1(b) shows the Poincaré map of the double-torus chaotic attractor on the $x-z$ plane. Its Lyapunov exponent spectrum is given by $L E_{1}=0.013, L E_{2}=0$, $L E_{3}=-0.030$, and $L E_{4}=-0.982$.

A novel fourth-order circuit has been designed for physically verifying the previous double-torus chaotic attractor. Fig. 2 shows the circuit diagram. This circuit diagram includes two main parts; that is, the first part shows the basic fourth-order double-torus circuit $N_{0}$; the second part shows the sign function generator in the $x$-direction $N_{1}$. All supply voltages and saturated voltages of operational amplifiers are $\pm 15 \mathrm{~V}$ and $V_{\text {sat }}=$ $\pm 13.5 \mathrm{~V}$, respectively. All resistances are exactly adjustable resistors or potentiometers. Here, $\tau=t / R_{0} C_{0}$ is the transformation factor of the time scale, in which $1 / R_{0} C_{0}$ is the integral constant of the integrator $N_{0}$. Also, $\alpha=R / R_{1}=R / R_{5}$. Then one can get the exact value of $\alpha$ by adjusting resistors $R_{1}$ and $R_{5}$.

It should be pointed out that one adds the capacitance $C 5$ with high capability $(2 u \mathrm{~F})$ in Fig. 2 for enhancing the coupling between subcircuits. Our experiments show that the trajectory can quickly and evenly run through the whole region of the double tori under the help of $C 5$. The main function of $C 5$ is to enhance the current distribution uniformity and prevent the longterm staying of the current in a local region. However, we can observe the same phenomena without $C 5$ for enough long time's observation.

Fig. 3 shows the experimental observation of the double-torus chaotic attractor.

\section{B. Dynamical Behaviors of the Double-Torus System}

In this subsection, the dynamical behaviors of the doubletorus system (1) are further investigated.

Obviously, system (1) has three equilibrium points: $E^{+}\left(A_{1}, 0,0,0\right), E^{O}(0,0,0,0)$, and $E^{-}\left(-A_{1}, 0,0,0\right)$. The corresponding Jacobian matrices and their characteristic equations of the zeros $E^{ \pm, O}$ are, respectively

$$
J=\left(\begin{array}{cccc}
0 & 1 & 0 & 0 \\
0 & 0 & 1 & 0 \\
0 & 0 & 0 & 1 \\
-\alpha & -\beta & -\gamma & -\delta
\end{array}\right)
$$




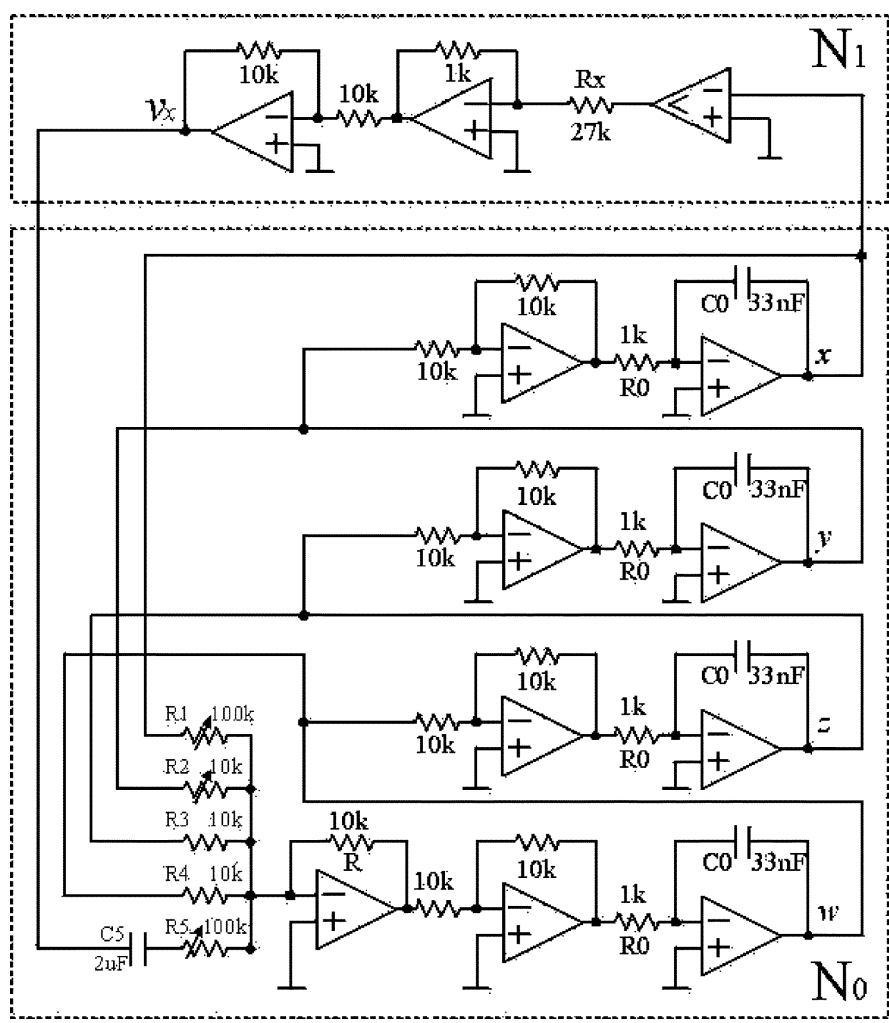

Fig. 2. Fourth-order double-torus circuit.

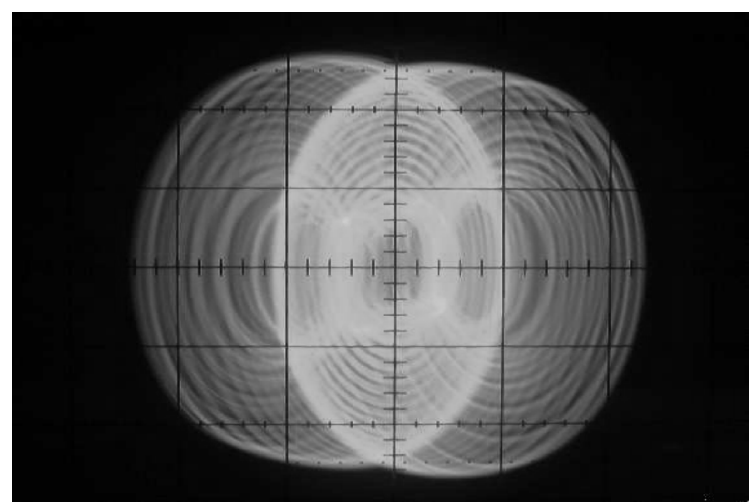

Fig. 3. Experimental observations of double-torus chaotic attractors, where $x=0.2 \mathrm{~V} / \mathrm{div}, y=0.1 \mathrm{~V} / \mathrm{div}$.

and

$$
\lambda^{4}+\delta \lambda^{3}+\gamma \lambda^{2}+\beta \lambda+\alpha=0
$$

Then, the four roots of (3) are given by

$$
\left\{\begin{array}{l}
\lambda_{1}=-\frac{1}{4} \delta+\frac{1}{2} R+\frac{1}{2} D=\mu_{1}+i \nu_{1} \\
\lambda_{2}=-\frac{1}{4} \delta+\frac{1}{2} R-\frac{1}{2} D=\mu_{2}+i \nu_{2} \\
\lambda_{3}=-\frac{1}{4} \delta-\frac{1}{2} R+\frac{1}{2} E=\mu_{3}+i \nu_{3} \\
\lambda_{4}=-\frac{1}{4} \delta-\frac{1}{2} R-\frac{1}{2} E=\mu_{4}+i \nu_{4}
\end{array}\right.
$$

where

$$
R=\sqrt{\frac{1}{4} \delta^{2}-\gamma+y_{1}}
$$

$$
\begin{aligned}
& D= \begin{cases}\sqrt{\frac{3}{4} \delta^{2}-R^{2}-2 \gamma+\frac{4 \delta \gamma-8 \beta-\delta^{3}}{4} R^{-1}}, & \text { for } R \neq 0 \\
\sqrt{\frac{3}{4} \delta^{2}-2 \gamma+2 \sqrt{y_{1}^{2}-4 \alpha},} & \text { for } R=0\end{cases} \\
& E= \begin{cases}\sqrt{\frac{3}{4} \delta^{2}-R^{2}-2 \gamma-\frac{4 \delta \gamma-8 \beta-\delta^{3}}{4}} R^{-1}, & \text { for } R \neq 0 \\
\sqrt{\frac{3}{4} \delta^{2}-2 \gamma-2 \sqrt{y_{1}^{2}-4 \alpha},} & \text { for } R=0\end{cases}
\end{aligned}
$$

in which $y_{1}$ is a real root of the equation

$$
y^{3}-\gamma y^{2}+(\beta \delta-4 \alpha) y+\left(4 \alpha \gamma-\beta^{2}-\delta^{2} \alpha\right)=0 \text {. }
$$

When $\alpha=0.1, A_{1}=0.5$, the three equilibrium points of system (1) are $E^{ \pm}( \pm 0.5,0,0,0)$ and $E^{O}(0,0,0,0)$ and their corresponding eigenvalues are $\lambda_{1,2} \approx 0.0276 \pm i 0.9760, \lambda_{3}=$ $-0.1111, \lambda_{4}=-0.9440$. That is, the three equilibrium points are saddle points with index 2.

Similarly, to generate multi-torus chaotic attractors from system (1), assume that

$$
\left\{\begin{array}{l}
\mu_{1}=\mu_{2}>0 \\
\nu_{1}=-\nu_{2}>0 \\
\mu_{3}<0, \nu_{3}=0 \\
\mu_{3} \neq \mu_{4}<0, \nu_{4}=0
\end{array}\right.
$$

That is, its linearized system evaluated at all equilibrium points has two negative eigenvalues and one pair of complex conjugate eigenvalues with positive real parts. Moreover, all equilibrium points are two-dimensionally unstable equilibrium points, called equilibrium points with index 2.

In addition, the state space of system (1) can be divided into three different subspaces

$$
\begin{aligned}
V_{+} & =\{\mathbf{X} \mid x>0\} \\
W_{O} & =\{\mathbf{X} \mid x=0\} \\
V_{-} & =\{\mathbf{X} \mid x<0\}
\end{aligned}
$$

where $\mathbf{X}=(x, y, z, w)^{T}$. System (1) has a natural symmetry under the coordinates transformation $(x, y, z, w) \rightarrow$ $(-x,-y,-z,-w)$, which persists for all parameter values. The variation of the volume $V(t)$ of a small element, $\delta V(t)=\delta x \delta y \delta z \delta w$ in the state space, is determined by the divergence of the flow

$$
\nabla V=\frac{\partial \dot{x}}{\partial x}+\frac{\partial \dot{y}}{\partial y}+\frac{\partial \dot{z}}{\partial z}+\frac{\partial \dot{w}}{\partial w}=-\delta<0 .
$$

That is, system (1) is dissipative in each subspace.

For subspaces $V_{ \pm}$and $W_{O}$, the dynamical equations are described by

$$
\left(\begin{array}{l}
\frac{d \bar{x}}{d \tau} \\
\frac{d \bar{y}}{d \tau} \\
\frac{d \bar{z}}{d \tau} \\
\frac{d \bar{w}}{d \tau}
\end{array}\right)=\left(\begin{array}{cccc}
0 & 1 & 0 & 0 \\
0 & 0 & 1 & 0 \\
0 & 0 & 0 & 1 \\
-\alpha & -\beta & -\gamma & -\delta
\end{array}\right)\left(\begin{array}{c}
\bar{x} \\
\bar{y} \\
\bar{z} \\
\bar{w}
\end{array}\right)
$$

where

$$
\begin{aligned}
& (\bar{x}, \bar{y}, \bar{z}, \bar{w})^{T}=\left(x-A_{1}, y, z, w\right)^{T}, \quad \text { for } \mathbf{X} \in V_{+} \\
& (\bar{x}, \bar{y}, \bar{z}, \bar{w})^{T}=(x, y, z, w)^{T}, \quad \text { for } \mathbf{X} \in W_{O} \\
& (\bar{x}, \bar{y}, \bar{z}, \bar{w})^{T}=\left(x+A_{1}, y, z, w\right)^{T}, \text { for } \quad \mathbf{X} \in V_{-} .
\end{aligned}
$$


Assume that (5) holds. The four eigenvalues of system (6) are $\mu_{j}+i \nu_{j}(1 \leq j \leq 4)$. Let their corresponding eigenvectors be $\eta_{1,2}=\left(e_{11}+i e_{12}, e_{21}+i e_{22}, e_{31}+i e_{32}, e_{41}+i e_{42}\right)^{T}, \eta_{3}=$ $\left(e_{13}, e_{23}, e_{33}, e_{43}\right)^{T}, \eta_{4}=\left(e_{14}, e_{24}, e_{34}, e_{44}\right)^{T}$, respectively. Thus, the exact solution of (6) is given by

$$
\begin{aligned}
&\left(\begin{array}{c}
\bar{x} \\
\bar{y} \\
\bar{z} \\
\bar{w}
\end{array}\right)= S_{1} e^{\mu_{1} \tau}\left(\begin{array}{l}
e_{11} \cos \nu \tau-e_{12} \sin \nu \tau \\
e_{21} \cos \nu \tau-e_{22} \sin \nu \tau \\
e_{31} \cos \nu \tau-e_{32} \sin \nu \tau \\
e_{41} \cos \nu \tau-e_{42} \sin \nu \tau
\end{array}\right) \\
&+S_{2} e^{\mu_{1} \tau}\left(\begin{array}{l}
e_{12} \cos \nu \tau+e_{11} \sin \nu \tau \\
e_{22} \cos \nu \tau+e_{21} \sin \nu \tau \\
e_{32} \cos \nu \tau+e_{31} \sin \nu \tau \\
e_{42} \cos \nu \tau+e_{41} \sin \nu \tau
\end{array}\right) \\
&+S_{3} e^{\mu_{3} \tau}\left(\begin{array}{l}
e_{13} \\
e_{23} \\
e_{33} \\
e_{43}
\end{array}\right)+S_{4} e^{\mu_{4} \tau}\left(\begin{array}{l}
e_{14} \\
e_{24} \\
e_{34} \\
e_{44}
\end{array}\right)
\end{aligned}
$$

where $S_{1}, S_{2}, S_{3}, S_{4}$ are real constants to be determined by the initial condition $\left(x_{0}, y_{0}, z_{0}, w_{0}\right)^{T}$.

As one can see in Section III, system (6) is also the basic generator of MDMT chaotic attractors.

\section{DESIGN OF MDMT CHAOTIC ATtRACTORS}

In this section, a systematic design approach is proposed for generating MDMT chaotic attractors based on the fourth-order double-torus chaotic circuit (1).

\section{A. A Fourth-Order MDMT Chaotic System}

We propose a novel fourth-order MDMT chaotic system, described by

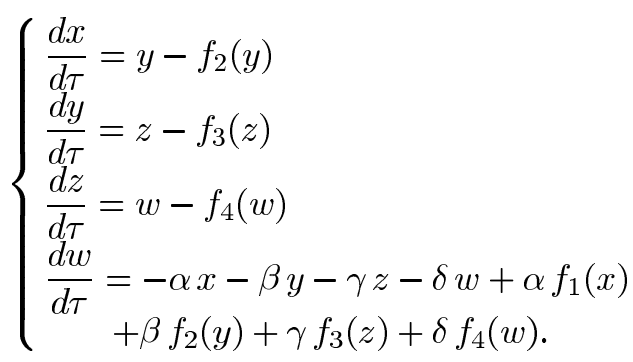

where $\alpha, \beta, \gamma, \delta$ are system parameters and $f_{1}(x), f_{2}(y), f_{3}(z), f_{4}(w)$ are controllers, which determine the number of tori in the $x, y, z, w$ directions, respectively.

To generate MDMT chaotic attractors from system (8), we introduces step function series or saturated function series into system (8). Assume that $f_{1}(x), f_{2}(y), f_{3}(z), f_{4}(w)$ are the step function series. That is

$$
f_{i}\left(\xi_{i}\right)=A_{i} \sum_{j=1}^{N_{i}}\left\{\operatorname{sgn}\left[\xi_{i}-(2 j-1) A_{i}\right]+\operatorname{sgn}\left[\xi_{i}+(2 j-1) A_{i}\right]\right\}
$$

or

$$
f_{i}\left(\xi_{i}\right)=A_{i} \sum_{j=0}^{M_{i}}\left[\operatorname{sgn}\left(\xi_{i}-2 j A_{i}\right)+\operatorname{sgn}\left(\xi_{i}+2 j A_{i}\right)\right]
$$

where $1 \leq i \leq 4, \xi_{1}=x, \xi_{2}=y, \xi_{3}=z, \xi_{4}=w, N_{i}$ and $M_{i}(1 \leq i \leq 4)$ are nonnegative integers, $\operatorname{sgn}(\cdot)$ is a sign function, and $A_{i}(1 \leq i \leq 4)$ are control parameters of the corresponding step function series with $A_{1}>0$ and $A_{2}=A_{3}=$ $A_{4}=A_{1} / 4$.

Similarly, suppose that $f_{1}(x), f_{2}(y), f_{3}(z), f_{4}(w)$ are saturated function series. That is

$$
\begin{aligned}
f_{i}\left(\xi_{i}\right)=\frac{A_{i}}{2 \eta} \sum_{\substack{j=-P_{i} \\
j \neq 0}}^{P_{i}}\{\mid & \mid\left[\xi_{i}-\left(2 j-\frac{|j|}{j}\right) A_{i}\right] \\
& \left.+\eta|-|\left[\xi_{i}-\left(2 j-\frac{|j|}{j}\right) A_{i}\right]-\eta \mid\right\}
\end{aligned}
$$

or

$$
f_{i}\left(\xi_{i}\right)=\frac{A_{i}}{2 \eta} \sum_{j=-Q_{i}}^{Q_{i}}\left[\left|\left(\xi_{i}-2 j A_{i}\right)+\eta\right|-\left|\left(\xi_{i}-2 j A_{i}\right)-\eta\right|\right]
$$

where $1 \leq i \leq 4, \xi_{1}=x, \xi_{2}=y, \xi_{3}=z, \xi_{4}=w, P_{i}$, and $Q_{i}(1 \leq i \leq 4)$ are nonnegative integers, $\eta=0.001$ and $A_{i}(1 \leq i \leq 4)$ are control parameters of the corresponding saturated function series with $A_{1}>0$ and $A_{2}=A_{3}=A_{4}=A_{1} / 4$. Moreover, the chaotic regions of parameter $\alpha$ is depending on the magnitudes of parameters $N_{i}$ and $M_{i}(1 \leq i \leq 4)$. That is, the larger the parameters $N_{i}$ and $M_{i}(1 \leq i \leq 4)$, the larger the parameters $\alpha, \beta, \gamma, \delta$. The bifurcation diagrams and Lyapunov exponent spectrum are also further analyzed, but omitted here for simplicity.

Remark 1: Obviously, system (8) with (9) [or (10), or (11), or (12)] becomes system (6) in every corresponding subspace via a simple coordinates transformation. Therefore, system (6) is the basic generator of the MDMT chaotic system (8) with (9) [or (10), or (11), or (12)] and plays a key role in chaos generation. In fact, according to the switching rules defined by $f_{i}\left(\xi_{i}\right)(1 \leq i \leq 4)$, system (8) can be divided into many small subspaces, $V_{(i, j, k, h)}^{(x, y, z, w)}$ and $V_{(i, j, k, h)}^{(x, I, y, J, z, K, w, H)}$. In each subspace, $V_{(i, j, k, h)}^{(x, y, z, w)}$ and $V_{(i, j, k, h)}^{(x, I, y, J, z, w, H)}$, system (8) can be transformed into the basic linear system (6) by the following linear transformation: $\bar{x}=x-f_{1}(x)$, $\bar{y}=y-f_{2}(y), \bar{z}=z-f_{3}(z), \bar{w}=w-f_{4}(w)$, where $(x, y, z, w)^{T} \in V_{(i, j, k, h)}^{(x, y, z, w)}$ or $V_{(i, j, k, h)}^{(x, I, y, J, z, K, w, H)}$.

\section{B. 1-D n-Torus Chaotic Attractors}

When $f_{1}(x) \neq 0, f_{2}(y)=f_{3}(z)=f_{4}(w)=0$, system (8) with (9) [or (10), or (11), or (12)] can generate 1-D multitorus chaotic attractors. In this subsection, we always assume that $f_{1}(x) \neq 0, f_{2}(y)=f_{3}(z)=f_{4}(w)=0$. In general, system (8) with (9) can create a $4 N_{1}$-torus chaotic attractor, and system (8) with (10) can generate a $\left(4 M_{1}+2\right)$-torus chaotic attractor. In particular, system (8) with (10) becomes system (1) for $M_{1}=0$.

Fig. 4(a) and (b) show the bifurcation diagram versus parameter $\alpha$ and the maximal Lyapunov exponent of system (8) with (9) for $N_{1}=4$ and $A_{1}=0.5$, respectively.

When $\alpha=0.1, \beta=\gamma=\delta=1, A_{1}=0.5, M_{1}=1$, system (8) with (10) has a 1-D 6-torus chaotic attractor as shown 


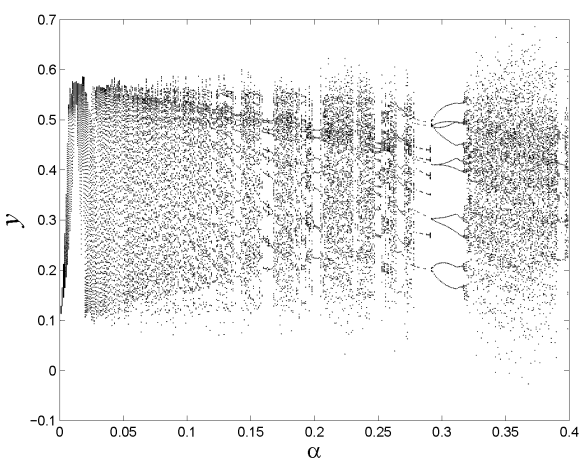

(a)

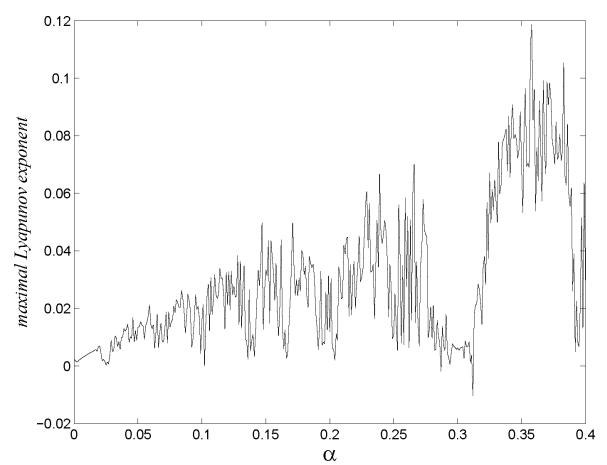

(b)

Fig. 4. (a) Bifurcation diagram versus parameter $\alpha$, where there exists a period-doubling bifurcation route to chaos. (b) Maximal Lyapunov exponent spectrum versus parameter $\alpha$.

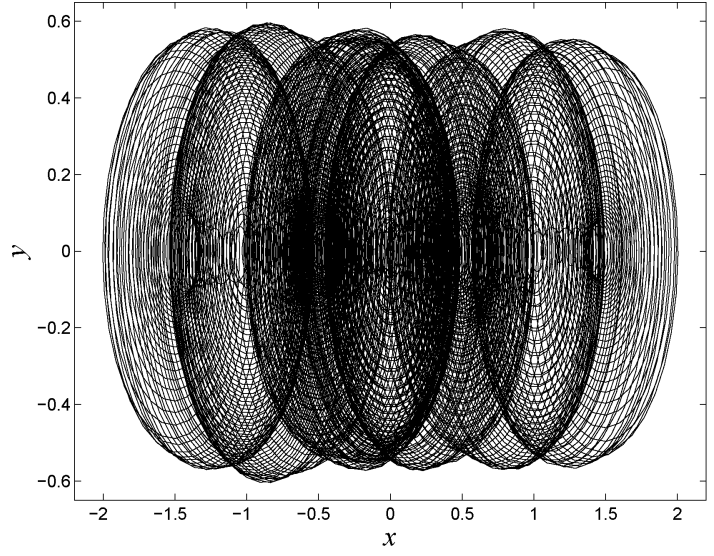

(a)

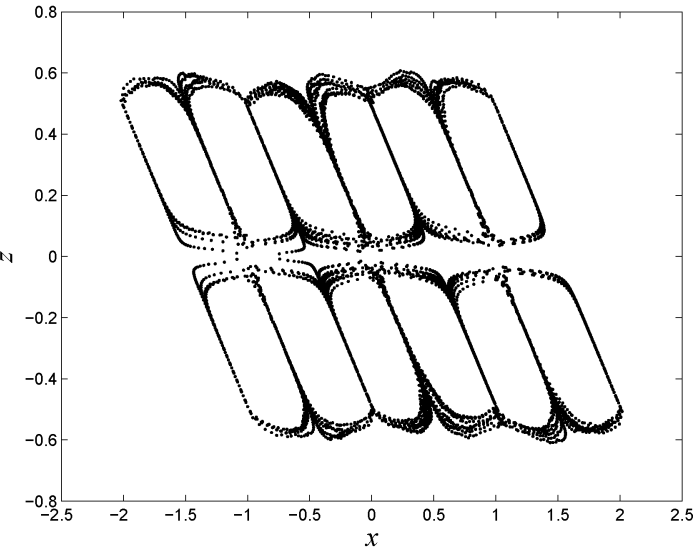

(b)

Fig. 5. 1-D 6-torus chaotic attractor. (a) $x-y$ plane projection. (b) Poincare map on the $x-z$ plane with $y=0$.

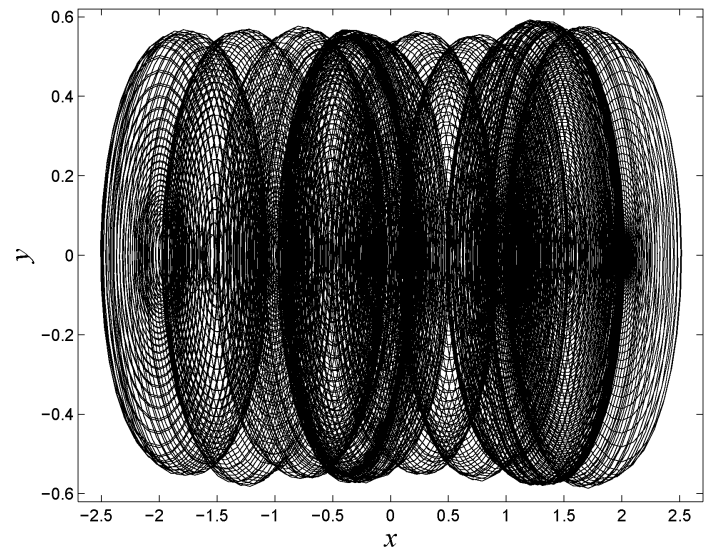

(a)

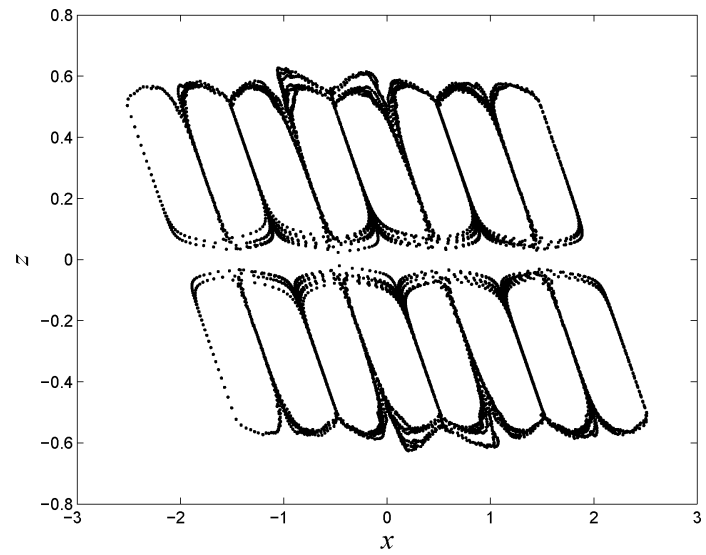

(b)

Fig. 6. 1-D 8-torus chaotic attractor. (a) $x-y$ plane projection. (b) Poincaré map on the $x-z$ plane with $y=0$.

in Fig. 5(a). Fig. 5(b) shows the Poincaré map of this 6-torus chaotic attractor on the $x-z$ plane.

Similarly, for $\alpha=0.1, \beta=\gamma=\delta=1, A_{1}=0.5, N_{1}=$ 2 , system (8) with (9) has a 1-D 8-torus chaotic attractor as shown in Fig. 6(a). Fig. 6(b) shows the poincaré mapping of this 8-torus chaotic attractor on $x-z$ plane.

Since $f_{1}(x) \neq 0, f_{2}(y)=f_{3}(z)=f_{4}(w)=0$, all equilibrium points of system (8) are $\left(x_{i}^{E}, 0,0,0\right)$ with $f_{1}\left(x_{i}^{E}\right)=x_{i}^{E}$. For example, when $\alpha=0.1, \beta=\gamma=\delta=1, A_{1}=0.5, N_{1}=$
2 , system (8) with (9) has 9 equilibrium points $\left(x_{i}^{E}, 0,0,0\right)$ with $x_{i}^{E} \in\{0, \pm 0.5, \pm 1, \pm 1.5, \pm 2\}$ as shown by "O" and " $\mathrm{X}$ " in Fig. 7. Our analysis shows that only equilibrium points "O" can generate tori, called step equilibrium points, while the equilibrium points " $x$ " can connect two neighboring tori, called critical equilibrium points. From Fig. 7, one can see that every outside step equilibrium point can only generate one torus. However, every insider step equilibrium point can create two tori. Therefore, the total number of tori is always an even number. In 


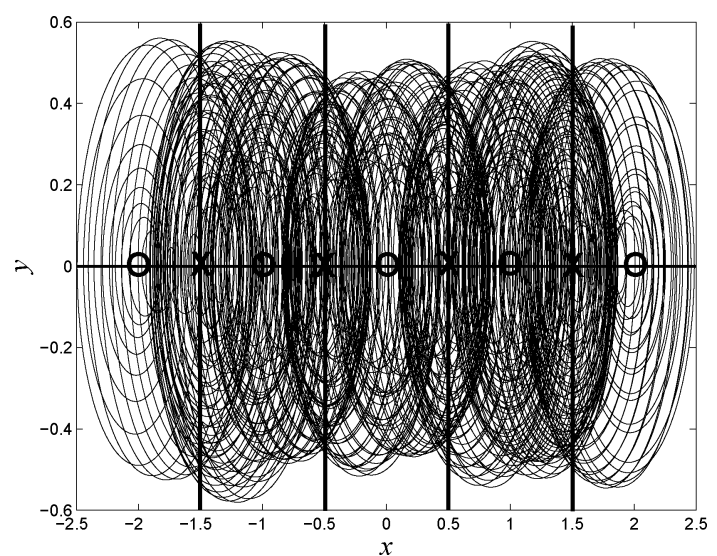

Fig. 7. Equilibrium points of 1-D 8-torus chaotic attractor.

particular, every step equilibrium point of the MDMT system can only generate one torus.

System (8) with (9) can be divided into $\left(4 N_{1}+1\right)$ subspaces

$$
\begin{aligned}
V_{-N_{1}}^{x}= & \left\{\mathbf{X} \mid x<\left(-2 N_{1}+1\right) A_{1}\right\} \\
V_{ \pm i}^{x}= & \left\{\mathbf{X} \mid( \pm 2 i-1) A_{1}<x<( \pm 2 i+1) A_{1}\right\} \\
& \quad\left(1 \leq i \leq N_{1}-1\right) \\
V_{0}^{x}= & \left\{\mathbf{X} \mid-A_{1}<x<A_{1}\right\} \\
V_{N_{1}}^{x}= & \left\{\mathbf{X} \mid x>\left(2 N_{1}-1\right) A_{1}\right\} \\
W_{ \pm i}^{x}= & \left\{\mathbf{X} \mid x=( \pm 2 i \mp 1) A_{1}\right\} \quad\left(1 \leq i \leq N_{1}\right)
\end{aligned}
$$

where $\mathbf{X}=(x, y, z, w)^{T}$. Subspaces $V_{i}^{x}\left(-N_{1} \leq i \leq N_{1}\right)$ and $W_{ \pm i}^{x}\left(1 \leq i \leq N_{1}\right)$ are called the step subspaces and critical subspaces in the $x$-direction, respectively. System (8) with (9) has $\left(4 N_{1}+1\right)$ equilibrium points: $\left(x_{i}^{E}, 0,0,0\right)$ with $x_{i}^{E}=\left\{-2 N_{1} A_{1},\left(-2 N_{1}+1\right) A_{1}, \ldots, 2 N_{1} A_{1}\right\}$. Every subspace has one and only one equilibrium point. However, only the step equilibrium points, $\left(x_{i}^{E}, 0,0,0\right)$ with $x_{i}^{E}=\left\{-2 N_{1} A_{1},-2\left(N_{1}-1\right) A_{1}, \ldots, 2 N_{1} A_{1}\right\}$, can generate torus. All critical equilibrium points, $\left(x_{i}^{E}, 0,0,0\right)$ with $x_{i}^{E}=\left\{\left(-2 N_{1}+1\right) A_{1},\left(-2 N_{1}+1\right) A_{1}, \ldots,\left(2 N_{1}-1\right) A_{1}\right\}$, cannot create torus.

In each subspace, $V_{i}^{x}$ or $W_{i}^{x}$, system (8) with (9) can be transformed into the basic linear system (6) by a coordinates transformation: $\bar{x}=x-f_{1}(x), \bar{y}=y, \bar{z}=z, \bar{w}=w$, with $\mathbf{X}=(x, y, z, w)^{T}$. However, system (8) with (10) can generate torus only in its step subspaces in the $x$-direction. Therefore, system (6) is the basic generator of the multi-torus chaotic system (8) with (9). System (8) with (9) creates complex dynamical behaviors via displacement and switching transformations based on the basic linear system (6). Similarly, system (8) with (10) [or (11), or (12)] generates complex dynamical behaviors via displacement and switching transformations based on the basic linear system (6).

Remark 2: Here the step equilibrium point (or subspace) and critical equilibrium point (or subspace) are not two proper nouns but two specific kinds of equilibria with different functions. In detail, the step equilibrium point can generate torus but the critical equilibrium point can connect two neighboring tori. The underlying theoretical mechanisms are also unclear and deserve further investigation in the near future.

\section{MDMT Chaotic Attractors}

System (8) with (9) [or (10), or (11), or (12)] can also generate various 2-D, 3-D, or 4-D multi-torus chaotic attractors. For simplification, we will briefly describe the 2-D and 3-D cases and then further discuss the 4-D case in the following.

When $f_{1}(x) \neq 0, f_{2}(y) \neq 0, f_{3}(z)=f_{4}(w)=0$, system (8) with (9) [or (10), or (11), or (12)] can create 2-D multitorus chaotic attractors. In general, system (8) with (9) or (10) can generate various 2-D $\pi_{1} \times \pi_{2}$-torus chaotic attractors for $f_{1}(x) \neq 0, f_{2}(y) \neq 0, f_{3}(z)=f_{4}(w)=0$, where $\pi_{i}=2 N_{i}+1$ or $\pi_{i}=2 M_{i}+2$ for $i=1,2$. For example, when $\alpha=0.1$, $\beta=\gamma=\delta=1, A_{1}=0.5, A_{2}=A_{1} / 4, N_{1}=N_{2}=6$, system (8) with (9) has a 2-D $13 \times 13$-torus chaotic attractor as shown in Fig. 8(a). Fig. 8(b) shows the Poincaré map of this 2 -D $13 \times 13$-torus chaotic attractor on the $x-y$ plane. Since $f_{1}(x) \neq 0, f_{2}(y) \neq 0, f_{3}(z)=f_{4}(w)=0$, all equilibrium points of system (8) are $\left(x_{i}^{E}, y_{i}^{E}, 0,0\right)$ with $f_{1}\left(x_{i}^{E}\right)=x_{i}^{E}$ and $f_{2}\left(y_{i}^{E}\right)=y_{i}^{E}$.

When $f_{1}(x) \neq 0, f_{2}(y) \neq 0, f_{3}(z) \neq 0, f_{4}(w)=0$, system (8) with (9) [or (10), or (11), or (12)] can create 3-D multi-torus chaotic attractors. In general, system (8) with (9) or (10) can generate various 3-D grid $\pi_{1} \times \pi_{2} \times \pi_{3}$-torus chaotic attractors for $f_{1}(x) \neq 0, f_{2}(y) \neq 0, f_{3}(z) \neq 0, f_{4}(w)=0$, where $\pi_{i}=2 N_{i}+1$ or $\pi_{i}=2 M_{i}+2$ for $i=1,2,3$. For example, when $\alpha=0.1, \beta=\gamma=\delta=1, A_{1}=0.5$, $A_{2}=A_{3}=A_{1} / 4, N_{1}=N_{2}=6, N_{3}=3$, system (8) with (9) has a 3-D $13 \times 13 \times 7$-torus chaotic attractor as shown in Fig. 9(a) and (b). Fig. 9(c) shows the Poincaré map of this 3 -D $13 \times 13 \times 7$-torus chaotic attractor on the $y-z$ plane. Since $f_{1}(x) \neq 0, f_{2}(y) \neq 0, f_{3}(z) \neq 0, f_{4}(w)=0$, all equilibrium points of system (8) are $\left(x_{i}^{E}, y_{i}^{E}, z_{i}^{E}, 0\right)$ with $f_{1}\left(x_{i}^{E}\right)=x_{i}^{E}$, $f_{2}\left(y_{i}^{E}\right)=y_{i}^{E}$, and $f_{3}\left(z_{i}^{E}\right)=z_{i}^{E}$.

When $f_{1}(x) \neq 0, f_{2}(y) \neq 0, f_{3}(z) \neq 0, f_{4}(w) \neq 0$, system (8) with (9) [or (10), or (11), or (12)] can create 4-D multitorus chaotic attractors. In the left parts of this subsection, we always assume that $f_{1}(x) \neq 0, f_{2}(y) \neq 0, f_{3}(z) \neq 0, f_{4}(w) \neq$ 0 . In general, system (8) with (9) or (10) can generate various 4-D $\pi_{1} \times \pi_{2} \times \pi_{3} \times \pi_{4}$-torus chaotic attractors, where $\pi_{i}=$ $2 N_{i}+1$ or $\pi_{i}=2 M_{i}+2$ for $i=1,2,3,4$. For example, when $\alpha=0.1, \beta=\gamma=\delta=1, A_{1}=0.5, A_{2}=A_{3}=$ $A_{4}=A_{1} / 4, N_{1}=N_{2}=6, N_{3}=N_{4}=3$, system (8) with (9) has a 4-D grid $13 \times 13 \times 7 \times 7$-torus chaotic attractor as shown in Fig. 10(a)-(c). Fig. 10(d) shows the Poincaré map of this $13 \times 13 \times 7 \times 7$-torus chaotic attractor on the $x-y$ plane.

Since $f_{1}(x) \neq 0, f_{2}(y) \neq 0, f_{3}(z) \neq 0, f_{4}(w) \neq 0$, all equilibrium points of system (8) are $\left(x_{i}^{E}, y_{i}^{E}, z_{i}^{E}, w_{i}^{E}\right)$ with $f_{1}\left(x_{i}^{E}\right)=x_{i}^{E}, f_{2}\left(y_{i}^{E}\right)=y_{i}^{E}, f_{3}\left(z_{i}^{E}\right)=z_{i}^{E}$ and $f_{4}\left(w_{i}^{E}\right)=w_{i}^{E}$. For example, when $\alpha=0.1, \beta=\gamma=\delta=1$, $A_{1}=1, A_{2}=A_{3}=A_{4}=A_{1} / 4, N_{1}=N_{2}=2, N_{3}=$ $N_{4}=1$, system (8) with (9) has $9 \times 9 \times 5 \times 5$ equilibrium points $\left(x_{i}^{E}, y_{i}^{E}, z_{i}^{E}, w_{i}^{E}\right)$ with $x_{i}^{E} \in\{0, \pm 1, \pm 2, \pm 3, \pm 4\}$, $y_{i}^{E} \in\{0, \pm 0.25, \pm 0.5, \pm 0.75, \pm 1\}, z_{i}^{E} \in\{0, \pm 0.25, \pm 0.5\}$, and $w_{i}^{E} \in\{0, \pm 0.25, \pm 0.5\}$.

Define $V_{-N_{4}}^{w}=\left\{\mathbf{X} \mid w<\left(-2 N_{4}+1\right) A_{4}\right\}$

$V_{ \pm h}^{w}=\left\{\mathbf{X} \mid( \pm 2 h-1) A_{4}<w<( \pm 2 h+1) A_{4}\right\}\left(1 \leq h \leq N_{4}-1\right)$ 


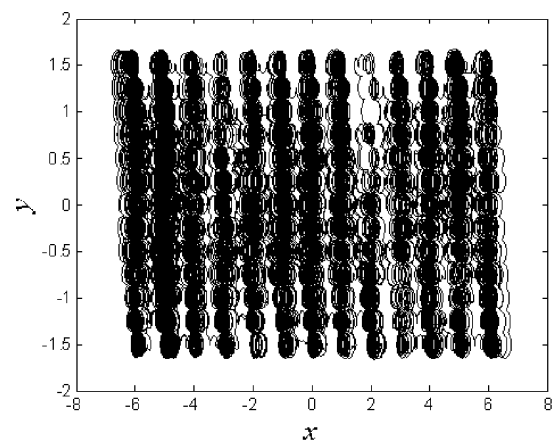

(a)

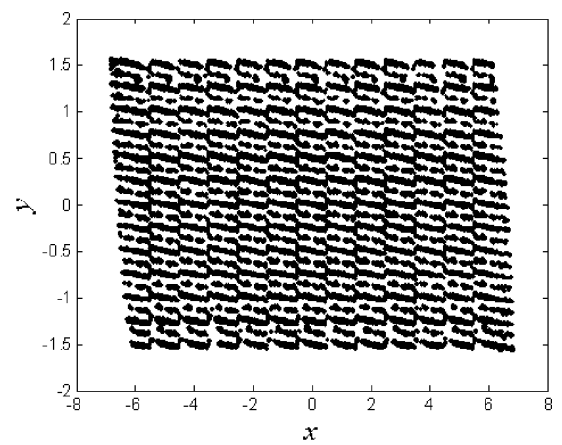

(b)

Fig. 8. 2-D $13 \times 13-$ torus chaotic attractor. (a) $x-y$ plane projection. (b) Poincaré map on the $x-y$ plane with $w=0$.

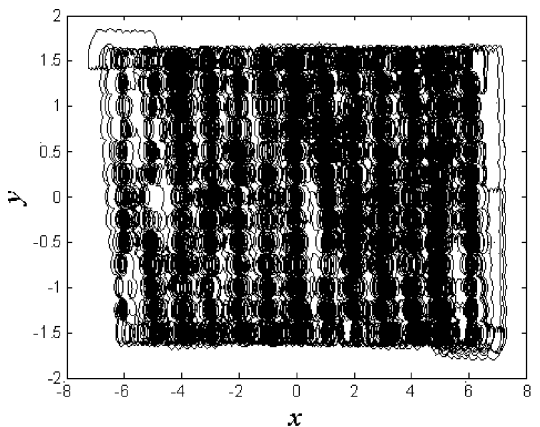

(a)

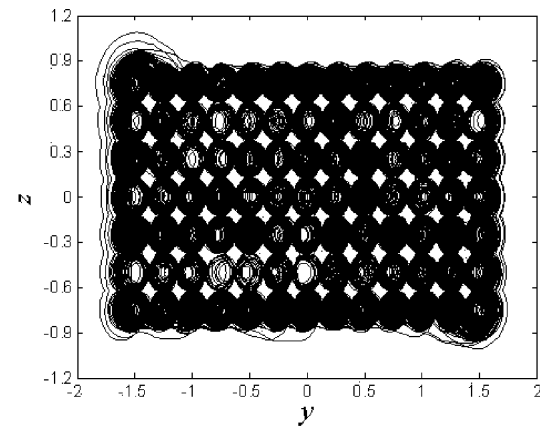

(b)

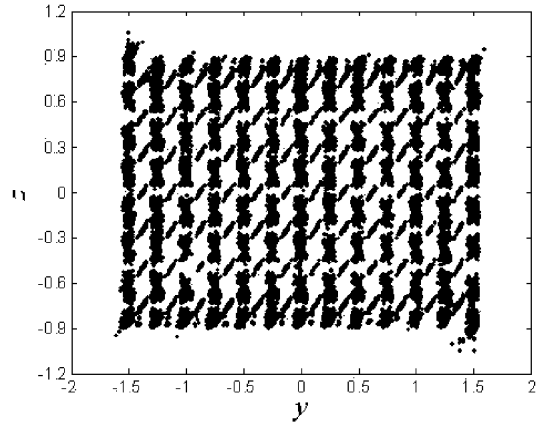

(c)

Fig. 9. 3-D $13 \times 13 \times 7$-torus chaotic attractor. (a) $x-y$ plane projection. (b) $y-z$ plane projection. (c) Poincaré map on the $y-z$ plane with $w=0$.

$V_{0}^{w}=\left\{\mathbf{X} \mid-A_{4}<w<A_{4}\right\}, V_{N_{4}}^{w}=\left\{\mathbf{X} \mid w>\left(2 N_{4}-1\right) A_{4}\right\}$, $W_{ \pm h}^{w}=\left\{\mathbf{X} \mid w=( \pm 2 h \mp 1) A_{4}\right\}\left(1 \leq h \leq N_{4}\right)$, where $\mathbf{X}=(x, y, z, w)^{T}$. Subspaces $V_{h}^{w}\left(-N_{4} \leq h \leq N_{4}\right)$ and $W_{+h}^{w}\left(1 \leq h \leq N_{4}\right)$ are called the step subspaces and critical subspaces in the $w$-direction, respectively. System (8) with (9) can be divided into $\left(4 N_{1}+1\right) \times\left(4 N_{2}+1\right) \times\left(4 N_{3}+1\right) \times\left(4 N_{4}+1\right)$ subspaces

$$
\begin{aligned}
& V_{(i, j, k, h)}^{(x, y, z)} \\
& =\left\{\mathbf{X} \mid x \in V_{i}^{x}, y \in V_{j}^{y}, z \in V_{k}^{z}, w \in V_{h}^{w}\right\} \\
& \quad\left(-N_{1} \leq i \leq N_{1},-N_{2} \leq j \leq N_{2},\right. \\
& \left.\quad-N_{3} \leq k \leq N_{3},-N_{4} \leq h \leq N_{4}\right) \\
& W_{(i, j, k, h)}^{(x, I, y, J, z, K, w, H)} \\
& =\left\{\mathbf{X} \mid x \in V_{i}^{(x, I)}, y \in V_{j}^{(y, J)}, z \in V_{k}^{(z, K)}, w \in V_{h}^{(w, H)}\right\}
\end{aligned}
$$

for $I=1, V_{i}^{(x, I)}=V_{i}^{x},-N_{1} \leq i \leq N_{1} ; I=2, V_{i}^{(x, I)}=$ $W_{i}^{x}, 0 \leq|i| \leq N_{1} ; J=1, V_{j}^{(y, J)}=V_{j}^{y},-N_{2} \leq j \leq N_{2}$;
$J=2, V_{j}^{(y, J)}=W_{j}^{y}, 0 \leq|j| \leq N_{2} ; K=1, V_{k}^{(z, K)}=V_{k}^{z}$, $-N_{3} \leq k \leq N_{3} ; K=2, V_{k}^{(z, K)}=W_{k}^{z}, 0 \leq|k| \leq N_{3} ; H=$ $1, V_{h}^{(w, H)}=V_{h}^{w},-N_{4} \leq h \leq N_{4} ; H=2, V_{h}^{(w, H)}=W_{h}^{w}$, $0 \leq|h| \leq N_{4}$, where $\mathbf{X}=(x, y, z, w)^{T}$. System (8) with (9) has $\left(4 N_{1}+1\right) \times\left(4 N_{2}+1\right) \times\left(4 N_{3}+1\right) \times\left(4 N_{4}+1\right)$ equilibrium points: $\left(x_{i}^{E}, y_{j}^{E}, z_{k}^{E}, w_{h}^{E}\right)$ with

$$
\begin{aligned}
x_{i}^{E} & =\left\{-2 N_{1} A_{1},\left(-2 N_{1}+1\right) A_{1}, \ldots, 2 N_{1} A_{1}\right\} \\
y_{j}^{E} & =\left\{-2 N_{2} A_{2},\left(-2 N_{2}+1\right) A_{2}, \ldots, 2 N_{2} A_{2}\right\} \\
z_{k}^{E} & =\left\{-2 N_{3} A_{3},\left(-2 N_{3}+1\right) A_{3}, \ldots, 2 N_{3} A_{3}\right\} \\
w_{h}^{E} & =\left\{-2 N_{4} A_{4},\left(-2 N_{4}+1\right) A_{4}, \ldots, 2 N_{4} A_{4}\right\} .
\end{aligned}
$$

Every subspace has one and only one equilibrium point. However, only the 4-D step equilibrium points, $\left(x_{i}^{E}, y_{j}^{E}, z_{k}^{E}, w_{h}^{E}\right)$ with

$$
\begin{aligned}
& x_{i}^{E}=\left\{-2 N_{1} A_{1},\left(-2 N_{1}+1\right) A_{1}, \ldots, 2 N_{1} A_{1}\right\} \\
& y_{j}^{E}=\left\{-2 N_{2} A_{2},\left(-2 N_{2}+1\right) A_{2}, \ldots, 2 N_{2} A_{2}\right\}
\end{aligned}
$$




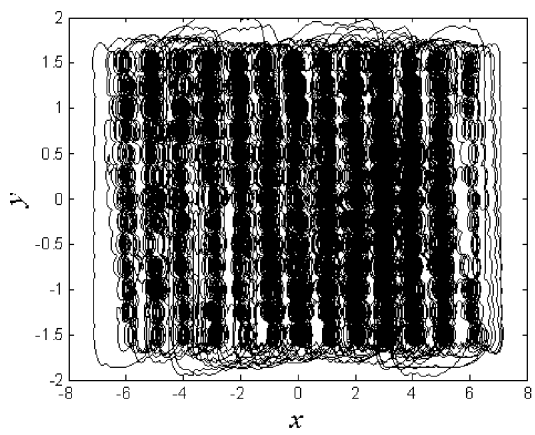

(a)

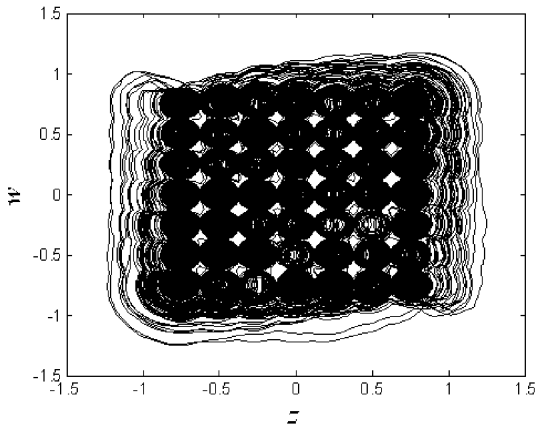

(c)

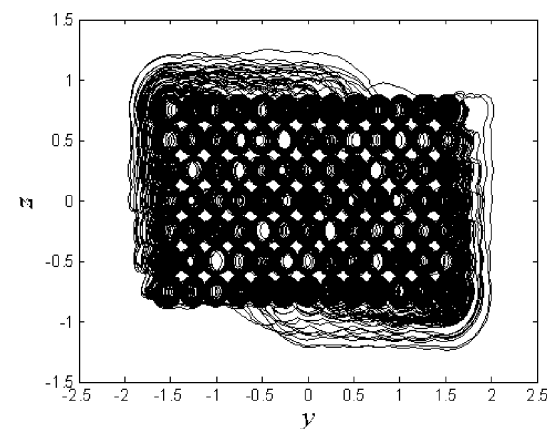

(b)

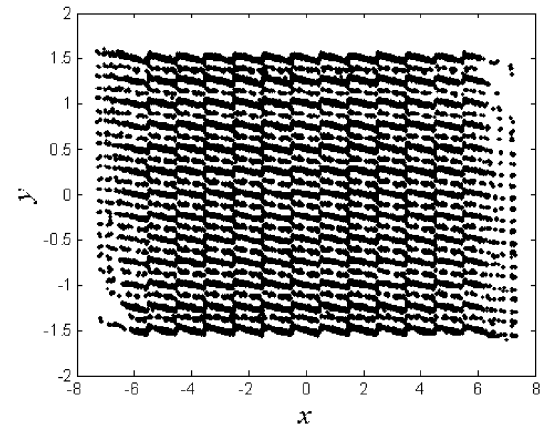

(d)

Fig. 10. 4-D $13 \times 13 \times 7 \times 7$-torus chaotic attractor. (a) $x-y$ plane projection. (b) $y-z$ plane projection. (c) $z-w$ plane projection. (d) Poincaré map on the $x-y$ plane with $w=0$.

$$
\begin{aligned}
z_{k}^{E} & =\left\{-2 N_{3} A_{3},\left(-2 N_{3}+1\right) A_{3}, \ldots, 2 N_{3} A_{3}\right\} \\
w_{h}^{E} & =\left\{-2 N_{4} A_{4},\left(-2 N_{4}+1\right) A_{4}, \ldots, 2 N_{4} A_{4}\right\}
\end{aligned}
$$

can generate torus. All 4-D critical equilibrium points, $\left(x_{i}^{E}, y_{j}^{E}, z_{k}^{E}, 0\right)$ with

$$
\begin{aligned}
x_{i}^{E} & =\left\{-2 N_{1} A_{1},\left(-2 N_{1}+1\right) A_{1}, \ldots, 2 N_{1} A_{1}\right\} \\
y_{j}^{E} & =\left\{-2 N_{2} A_{2},\left(-2 N_{2}+1\right) A_{2}, \ldots, 2 N_{2} A_{2}\right\} \\
z_{k}^{E} & =\left\{-2 N_{3} A_{3},\left(-2 N_{3}+1\right) A_{3}, \ldots, 2 N_{3} A_{3}\right\} \\
w_{h}^{E} & =\left\{-2 N_{4} A_{4},\left(-2 N_{4}+1\right) A_{4}, \ldots, 2 N_{4} A_{4}\right\}
\end{aligned}
$$

but

$$
\begin{aligned}
x_{i}^{E} & =\left\{-2 N_{1} A_{1},-2\left(N_{1}-1\right) A_{1}, \ldots, 2 N_{1} A_{1}\right\} \\
y_{j}^{E} & =\left\{-2 N_{2} A_{2},-2\left(N_{2}-1\right) A_{2}, \ldots, 2 N_{2} A_{2}\right\} \\
z_{k}^{E} & =\left\{-2 N_{3} A_{3},-2\left(N_{3}-1\right) A_{3}, \ldots, 2 N_{3} A_{3}\right\} \\
w_{h}^{E} & =\left\{-2 N_{4} A_{4},-2\left(N_{4}-1\right) A_{4}, \ldots, 2 N_{4} A_{4}\right\}
\end{aligned}
$$

cannot create torus.

In each subspace, $V_{(i, j, k, h)}^{(x, y, z, w)}$ or $W_{(i, j, k, h)}^{(x, I, y, J, z, K, w, H)}$, system (8) with (9) can be transformed into the basic linear system (6) by the following linear transformation: $\bar{x}=x-f_{1}(x), \bar{y}=y-$ $f_{2}(y), \bar{z}=z-f_{3}(z), \bar{w}=w-f_{4}(w)$ with $\mathbf{X}=(x, y, z, w)^{T}$. However, system (8) with (9) can create torus only in 4-D step subspaces

$$
\begin{array}{r}
V_{(i, j, k, h)}^{(x, y, z, w)}\left(-N_{1} \leq i \leq N_{1},-N_{2} \leq j \leq N_{2},-N_{3} \leq k \leq N_{3}\right. \\
\left.-N_{4} \leq h \leq N_{4}\right)
\end{array}
$$

Therefore, system (6) is the basic generator of the multi-torus chaotic system (8) with (9). System (8) with (9) creates complex dynamical behaviors via displacement and switching transformations based on the basic linear system (6). Similarly, system (8) with (10) [or (11), or (12)] generates complex dynamical behaviors via displacement and switching transformations based on the basic linear system (6).

\section{CiRCUIT IMPLEMENTATION OF MDMT CHAOTIC ATTRACTORS}

Based on the operational principles of the MDMT chaotic attractors, from (8)-(10), we have constructed a circuit diagram to physically realize various MDMT chaotic attractors.

Fig. 11 shows such a circuit diagram. This circuit diagram includes six different parts; that is, Part I: basic fourth-order multi-torus circuit $N_{0}$; Part II: generator $N_{1}$ of step function series in the $x$-direction; Part III: generator $N_{2}$ of step function series in the $y$-direction; Part IV: generator $N_{3}$ of step function series in the z-direction; Part V: generator $N_{4}$ of step function series in the w-direction; Part VI: switch linkages, including $K_{1}, K_{2}, K_{3}, K_{10}, K_{11}, K_{12}, K_{13}, K_{14}, K_{15}, K_{22}$. Assume that the supply voltages and saturated voltages of all operational amplifiers are $V= \pm 15 \mathrm{~V}$ and $V_{\text {sat }}= \pm 13.5 \mathrm{~V}$, respectively. Furthermore, switch linkages $K_{1}, K_{2}, K_{3}$ control the number of directions for the fourth-order MDMT chaotic attractors based on the basic fourth-order multi-torus circuit $N_{0}$ and capacitance coupling. On the other hand, switch linkages $K_{10}, K_{11}, K_{12}, K_{13}, K_{14}, K_{15}$ control the number of tori in the $x$-direction and switch linkage $K_{22}$ controls the number of tori in the $y$-direction for the fourth-order 


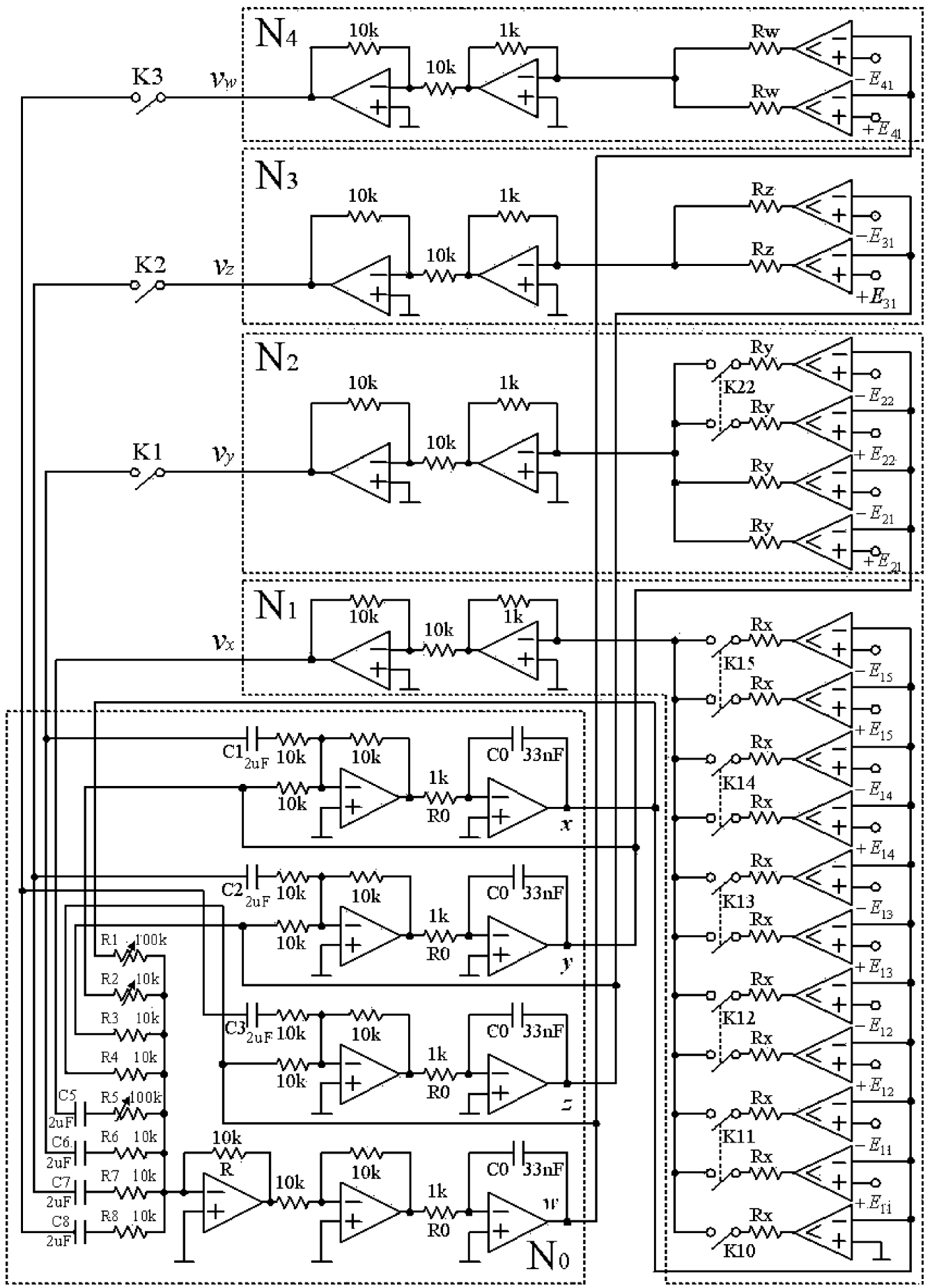

Fig. 11. Circuit diagram for implementing multidirectional grid multi-torus chaotic attractors.

MDMT chaotic attractors based on the generators $N_{1}$ and $N_{2}$, respectively.

According to the circuit diagram shown in Fig. 11, the state equation of the circuit in the integral form is described by

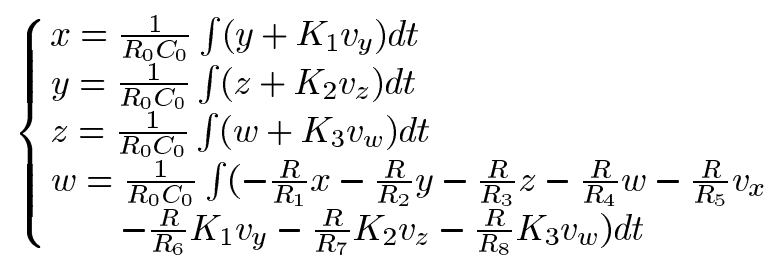

where $v_{x}, v_{y}, v_{z}, v_{w}$ are the step function series in the $x, y, z, w$-direction, respectively, given by

$$
\left\{\begin{aligned}
v_{x}= & -\frac{\left|V_{\text {sat }}\right|}{R_{x}}\left\{K_{10} \operatorname{sgn}(x)\right. \\
& \left.+\sum_{j=1}^{5} K_{1 j}\left[\operatorname{sgn}\left(x-E_{1 j}\right)+\operatorname{sgn}\left(x+E_{1 j}\right)\right]\right\} \\
v_{y}= & -\frac{\left|V_{s a t}\right|}{R_{y}} \sum_{j=1}^{2} K_{2 j}\left[\operatorname{sgn}\left(y-E_{2 j}\right)+\operatorname{sgn}\left(y+E_{2 j}\right)\right] \\
v_{z}= & -\frac{\left|V_{\text {sat }}\right|}{R_{z}} K_{31}\left[\operatorname{sgn}\left(z-E_{31}\right)+\operatorname{sgn}\left(z+E_{31}\right)\right] \\
v_{w}= & -\frac{\left|V_{\text {sat }}\right|}{R_{w}} K_{41}\left[\operatorname{sgn}\left(w-E_{41}\right)+\operatorname{sgn}\left(w+E_{41}\right)\right] .
\end{aligned}\right.
$$




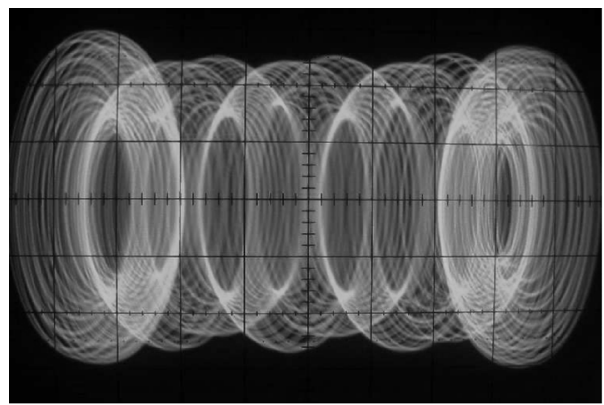

(a)

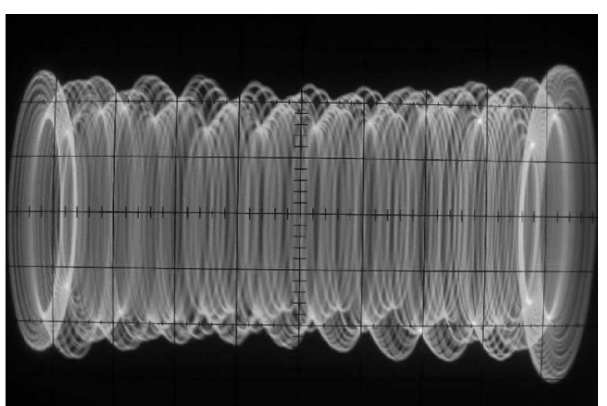

(b)

Fig. 12. Experimental observations of 1-D multi-torus chaotic attractors in the $x-y$ plane. (a) 8 -torus, where $x=0.25 \mathrm{~V} / \mathrm{div}, y=0.1 \mathrm{~V} / \mathrm{div}$. (b) 20 -torus, where $x=0.55 \mathrm{~V} / \mathrm{div}, y=0.1 \mathrm{~V} / \mathrm{div}$

Hereafter, $R_{0}=1 \mathrm{k} \Omega$ and $C_{0}=33 \mathrm{nF}$ are the integral resistor and capacitance, respectively. $R_{x}, R_{y}, R_{z}, R_{w}$ are the voltage-current conversion resistors. $V_{\text {sat }}= \pm 13.5 \mathrm{~V}$ are the saturated voltages of all operational amplifiers. $E_{1 j}(1 \leq j \leq$ $5), E_{2 k}(k=1,2), E_{31}, E_{41}$ are the step voltages of step function series. $\left(R / R_{i}\right)(1 \leq i \leq 8)$ are the transformation coefficients of the anti-summator, where $R=10 \mathrm{k} \Omega$ and $R_{i}(1 \leq$ $i \leq 8)$ are given in Fig. 11. $K_{21}=K_{31}=K_{41}=1$. $K_{1 j}(0 \leq j \leq 5), K_{i}(1 \leq i \leq 3), K_{22}$ are the switch functions, which are defined as 1 for switching on and 0 for switching off.

From (13) and (14), one gets

$$
\left\{\begin{aligned}
\frac{d x}{d \tau}= & y-K_{1} f_{2}(y) \\
\frac{d y}{d \tau}= & z-K_{2} f_{3}(z) \\
\frac{d z}{d \tau} & =w-K_{3} f_{4}(w) \\
\frac{d w}{d \tau} & =-\frac{R}{R_{1}} x-\frac{R}{R_{2}} y-\frac{R}{R_{3}} z-\frac{R}{R_{4}} w+\frac{R}{R_{5}} f_{1}(x) \\
& +\frac{R}{R_{6}} K_{1} f_{2}(y)+\frac{R}{R_{7}} K_{2} f_{3}(z)+\frac{R}{R_{8}} K_{3} f_{4}(w)
\end{aligned}\right.
$$

where $R / R_{1}=R / R_{5}=\alpha, R / R_{2}=R / R_{6}=\beta, R / R_{3}=$ $R / R_{7}=\gamma, R / R_{4}=R / R_{8}=\delta$, and

$$
\left\{\begin{aligned}
f_{1}(x)= & \frac{\left|V_{\text {sat }}\right|}{R_{x}}\left\{K_{10} \operatorname{sgn}(x)\right. \\
& \left.+\sum_{j=1}^{5} K_{1 j}\left[\operatorname{sgn}\left(x-E_{1 j}\right)+\operatorname{sgn}\left(x+E_{1 j}\right)\right]\right\} \\
f_{2}(y)= & \frac{\left|V_{\text {sat }}\right|}{R_{y}} \sum_{j=1}^{2} K_{2 j}\left[\operatorname{sgn}\left(y-E_{2 j}\right)+\operatorname{sgn}\left(y+E_{2 j}\right)\right] \\
f_{3}(z)= & \frac{\left|V_{\text {sat }}\right|}{R_{z}} K_{31}\left[\operatorname{sgn}\left(z-E_{31}\right)+\operatorname{sgn}\left(z+E_{31}\right)\right] \\
f_{4}(w) & =\frac{\left|V_{\text {sat }}\right|}{R_{w}} K_{41}\left[\operatorname{sgn}\left(w-E_{41}\right)+\operatorname{sgn}\left(w+E_{41}\right)\right] .
\end{aligned}\right.
$$

Here, $R_{i}(1 \leq i \leq 8)$ are exactly adjustable resistors. In practice, one can change system parameters $\alpha, \beta, \gamma, \delta$ by adjusting a set of resistors, $R_{1}, R_{5}, R_{2}, R_{6}, R_{3}, R_{7}, R_{4}$, and $R_{8}$. Moreover, $1 / R_{0} C_{0}$ is the integrator constant of the integrator $N_{0}$ shown in Fig. 11, and it is also the transformation factor of the time scale satisfying $\tau=t / R_{0} C_{0}$. In addition, one can change the distribution region of the frequency spectrum for a chaotic signal by tuning integral resistor $R_{0}$ or integral capacitance $C_{0}$. That is, when $R_{0}$ (or $C_{0}$ ) is decreasing, one can adjust the distribution region of the frequency spectrum of the chaotic signal to the high-frequency end. However, when $R_{0}$ (or $C_{0}$ ) is
TABLE I

ON-OFF OF SWITCHES AND THE NUMBER OF 1-D TORI

\begin{tabular}{c|c|c|c|c|c|c|c|c|c|c}
\hline$K_{1}$ & $K_{2}$ & $K_{3}$ & $K_{10}$ & $K_{11}$ & $K_{12}$ & $K_{13}$ & $K_{14}$ & $K_{15}$ & $K_{22}$ & $n$ \\
\hline off & off & off & on & off & off & off & off & off & off & $1 \sim 2$ \\
\hline off & off & off & on & on & off & off & off & off & off & 6 \\
\hline off & off & off & off & on & on & off & off & off & off & 8 \\
\hline off & off & off & off & on & on & on & off & off & off & 12 \\
\hline off & off & off & off & on & on & on & on & off & off & 16 \\
\hline off & off & off & off & on & on & on & on & on & off & 20 \\
\hline
\end{tabular}

TABLE II

CirCuIT PARAMETERS OF 1-D MULTI-TORUS ATTRACTORS

\begin{tabular}{c|c|c|c|c|c|c}
\hline$R_{x}(k \Omega)$ & $\pm E_{11}(V)$ & $\pm E_{12}(V)$ & $\pm E_{13}(V)$ & $\pm E_{14}(V)$ & $\pm E_{15}(V)$ & $n$ \\
\hline 54 & & & & & & $1 \sim 2$ \\
\hline 54 & \pm 0.50 & & & & & 6 \\
\hline 54 & \pm 0.25 & \pm 0.75 & & & & 8 \\
\hline 54 & \pm 0.25 & \pm 0.75 & \pm 1.25 & & & 12 \\
\hline 54 & \pm 0.25 & \pm 0.75 & \pm 1.25 & \pm 1.75 & & 16 \\
\hline 54 & \pm 0.25 & \pm 0.75 & \pm 1.25 & \pm 1.75 & \pm 2.25 & 20 \\
\hline
\end{tabular}

TABLE III

ON-OFF OF SWITCHES AND THE NUMBER OF 2-D MULTI-TORUS ATtRACTORS

\begin{tabular}{c|c|c|c|c|c|c|c|c|c|c}
\hline$K_{1}$ & $K_{2}$ & $K_{3}$ & $K_{10}$ & $K_{11}$ & $K_{12}$ & $K_{13}$ & $K_{14}$ & $K_{15}$ & $K_{22}$ & $n \times m$ \\
\hline on & off & off & off & on & off & off & off & off & off & $3 \times 5$ \\
\hline on & off & off & off & on & on & off & off & off & on & $5 \times 5$ \\
\hline
\end{tabular}

TABLE IV

Circuit Parameters of 2-D Multi-Torus ATtractors

\begin{tabular}{c|c|c|c|c|c|c}
\hline$R_{x}(k \Omega)$ & $R_{y}(k \Omega)$ & $\pm E_{11}(V)$ & $\pm E_{12}(V)$ & $\pm E_{21}(V)$ & $\pm E_{22}(V)$ & $n \times m$ \\
\hline 13.5 & 54 & \pm 1.00 & & \pm 0.25 & & $3 \times 5$ \\
\hline 13.5 & 54 & \pm 1.00 & \pm 3.00 & \pm 0.25 & \pm 0.75 & $5 \times 5$ \\
\hline
\end{tabular}

increasing, one can tune the distribution region of the frequency spectrum of the chaotic signal to the low-frequency end.

From the circuit diagram Fig. 11 and Tables I and II, one can get 1-D 1-, 2-, 6-, 8-, 12-, 16-, 20-torus chaotic attractors. Fig. 12 shows the experimental observations of the 8- and 20-torus chaotic attractors.

According to the circuit diagram shown in Fig. 11 and Tables III and IV, one can get 2-D $3 \times 5$ and $5 \times 5$ torus chaotic attractors as shown in Fig. 13.

Suppose that $K_{1}, K_{2}, K_{3}, K_{11}, K_{12}, K_{22}$ are switched on and $K_{10}, K_{13}, K_{14}, K_{15}$ are switched off. Then, the circuit diagram can generate a 3-D $5 \times 5 \times 3 \times 3$ torus chaotic attractor as shown in Fig. 14, with circuit parameters $R_{x}=13.5 \mathrm{k} \Omega, R_{y}=$ $R_{z}=R_{w}=54 \mathrm{k} \Omega, \pm E_{11}= \pm 1.00 \mathrm{~V} \pm E_{12}= \pm 3.00 \mathrm{~V}$, $\pm E_{21}= \pm E_{31}= \pm E_{41}= \pm 0.25 \mathrm{~V}, \pm E_{22}= \pm 0.75 \mathrm{~V}$.

Remark 3: Similarly, one can experimentally verify 3-D multi-torus chaotic attractors by switching the switcher linkages in Fig. 11. For simplicity, all experimental descriptions 


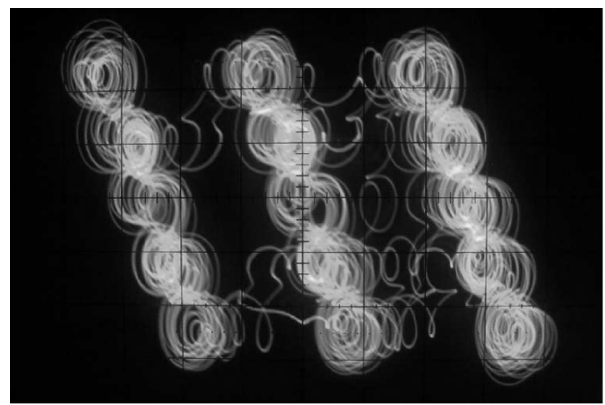

(a)

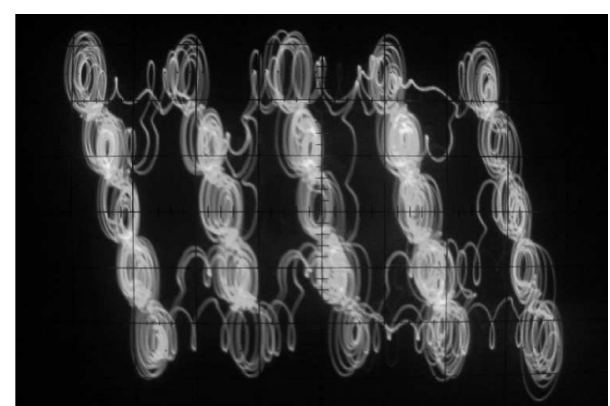

(b)

Fig. 13. Experimental observations of 2 -D multi-torus chaotic attractors in $x-y$ plane. (a) $3 \times 5$ torus, where $x=0.36 \mathrm{~V} /$ div, $y=0.2 \mathrm{~V} /$ div. (b) $3 \times 5$ torus, where $x=0.6 \mathrm{~V} / \mathrm{div}, y=0.2 \mathrm{~V} / \mathrm{div}$.

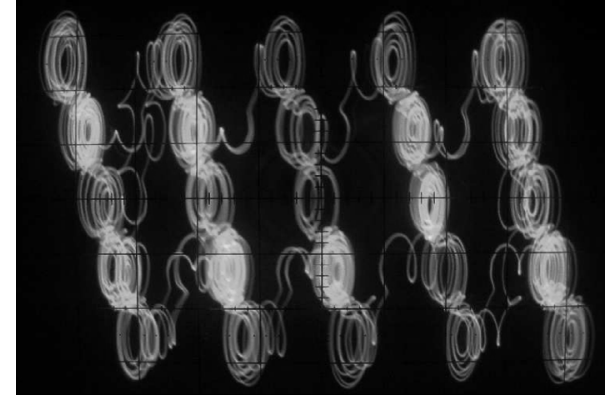

(a)

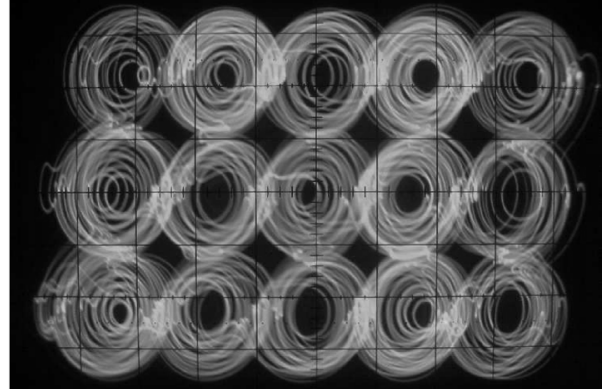

(b)

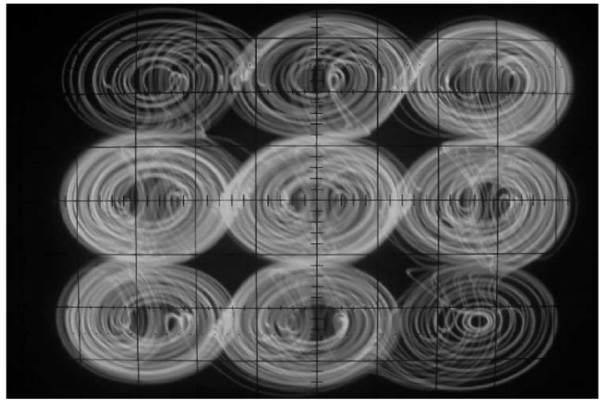

(c)

Fig. 14. Experimental observations of 4-D $5 \times 5 \times 3 \times 3$-torus chaotic attractors. (a) the $x-y$ plane, where $x=0.6 \mathrm{~V} /$ div, $y=0.2 \mathrm{~V} /$ div. (b) $y-z$ plane, where $x=0.16 \mathrm{~V} / \mathrm{div}, y=0.1 \mathrm{~V} / \mathrm{div}$. (c) $z-w$ plane, where $x=0.12 \mathrm{~V} / \mathrm{div}, y=0.12 \mathrm{~V} / \mathrm{div}$.

and observations are omitted here. In particular, to enhance the coupling between sub-circuits, one adds the capacitances $C 1, C 2, C 3, C 5, C 6, C 7, C 8$ with high capability $(2 u F)$ in Fig. 11. Our experiments show that the trajectory can quickly and evenly run through the whole region of all tori under the help of $C 1, C 2, C 3, C 5, C 6, C 7, C 8$. The main functions of these capacitances are to enhance the current distribution uniformity and prevent the longterm staying of the current in a local region. However, one can also observe these phenomena without these capacitances.

\section{CONCLUSION}

We have proposed a novel fourth-order double-torus chaotic circuit. By slightly modifying this basic circuit, a systematic theoretical design approach has been presented for creating various 1-D $n$-torus, 2-D $n \times m$-torus, 3-D $n \times m \times l$-torus, and 4-D $n \times m \times l \times p$-torus chaotic attractors. Some typical dynamical behaviors, including equilibrium points, bifurcations, Lyapunov exponents, and Poincaré maps, have been further investigated for the MDMT chaotic system. Our theoretical analysis shows that the MDMT chaotic attractors can be created by switching and displacing a basic linear circuit.

It should be pointed out that this is the first autonomous circuit reported in the literature for generating MDMT chaotic attractors. Also, this is the first time in the literature to physically implement a maximal 1-D 20-torus, a maximal 2-D $5 \times 5$ torus, and a maximal 4-D $5 \times 5 \times 3 \times 3$ torus chaotic attractors. We have also noticed various complex dynamic mechanisms, including very rich bifurcation phenomena, which deserve further investiagation in the near future.

\section{REFERENCES}

[1] S. M. Yu, J. Lü, and G. Chen, "Multifolded torus chaotic attractors: Design and implementation," Chaos, vol. 17, no. 1, p. 013118, Mar. 2007.

[2] M. E. Yalcin, J. A. K. Suykens, and J. Vandewalle, Cellular Neural Networks, Multi-Scroll Chaos and Synchronization. Singapore: World Scientific, 2005. 
[3] J. Lü and G. Chen, "Generating multiscroll chaotic attractors: Theories, methods and applications," Int. J. Bifurc. Chaos, vol. 16, no. 4, pp. 775-858, Apr. 2006.

[4] S.Özoğuz, A. S. Elwakil, and K. N. Salama, " $N$-scroll chaos generator using nonlinear transconductor," Electron. Lett., vol. 38, no. 14, pp. 685-686, Jul. 2002.

[5] A. S. Elwakil, "Integrator-based circuit-independent chaotic oscillator structure," Chaos, vol. 14, no. 2, pp. 364-369, Jun. 2004.

[6] A. E. Elwakil, "Canonical 4th order equations for chaos generation," in Proc. 9th Workshop Nonlinear Dyn. Electron. Syst. (NDES), 2001, pp. 181-184.

[7] J. A. K. Suykens, A. Huang, and L. O. Chua, "A family of $n$-scroll attractors from a generalized Chua's circuit," Int. J. Electron. Commun., vol. 51, no. 3, pp. 131-138, 1997.

[8] J. A. K. Suykens and J. Vandewalle, "Quasilinear approach to nonlinear systems and the design of $n$-double scroll ( $n=1,2,3,4, \ldots)$, , $G$, IEE Proc., vol. 138, no. 5, pp. 595-603, Oct. 1991.

[9] J. A. K. Suykens and J. Vandewalle, "Generation of $n$-double scrolls $(n=1,2,3,4, \ldots)$," IEEE Trans. Circuits Syst. I, Fundam. Theory Appl., vol. 40, no. 11, pp. 861-867, Nov. 1993.

[10] J. A. K. Suykens and L. O. Chua, " $n$-double scroll hypercubes in 1-D CNNs," Int. J. Bifurc. Chaos, vol. 7, no. 8, pp. 1873-1885, Aug. 1997.

[11] M. A. Aziz-Alaoui, "Differential equations with multispiral attractors," Int. J. Bifurcation Chaos, vol. 9, no. 6, pp. 1009-1039, Jun. 1999.

[12] M. E. Yalcin, S. Ozoguz, J. A. K. Suykens, and J. Vandewalle, " $n$-scroll chaos generators: A simple circuit model," Electron. Lett., vol. 37, no. 3, pp. 147-148, Feb. 2001.

[13] K. S. Tang, G. Q. Zhong, G. Chen, and K. F. Man, "Generation of $n$-scroll attractors via sine function," IEEE Trans. Circuits Syst. I, Fundam. Theory Appl., vol. 48, no. 11, pp. 1369-1372, Nov. 2001.

[14] G. Zhong, K. F. Man, and G. Chen, "A systematic approach to generating $n$-scroll attractors," Int. J. Bifurc. Chaos, vol. 12, no. 12, pp. 2907-2915, Dec. 2002.

[15] J. Lü, T. Zhou, G. Chen, and X. Yang, "Generating chaos with a switching piecewise-linear controller," Chaos, vol. 12, no. 2, pp. 344-349, Jun. 2002.

[16] J. Lü, X. Yu, and G. Chen, "Generating chaotic attractors with multiple merged basins of attraction: A switching piecewise-linear control approach," IEEE Trans. Circuits Syst. I, Fundam. Theory Appl., vol. 50, no. 2, pp. 198-207, Feb. 2003.

[17] M. E. Yalcin, J. A. K. Suykens, J. Vandewalle, and S. Ozoguz, "Families of scroll grid attractors," Int. J. Bifurc. Chaos, vol. 12, no. 1, pp. 23-41, Jan. 2002.

[18] F. Han, J. Lü, X. Yu, G. Chen, and Y. Feng, "Generating multi-scroll chaotic attractors via a linear second-order hysteresis system," Dyn. Continuous, Discrete Impulsive Syst. Ser. B: Appl. Algorithms, vol. 12, no. 1, pp. 95-110, 2005.

[19] J. Lü, F. Han, X. Yu, and G. Chen, "Generating 3-D multi-scroll chaotic attractors: A hysteresis series switching method," Automatica, vol. 40, no. 10 , pp. 1677-1687, Oct. 2004.

[20] J. Lü, G. Chen, X. Yu, and H. Leung, "Design and analysis of multiscroll chaotic attractors from saturated function series," IEEE Trans. Circuits Syst. I, Reg. Papers, vol. 51, no. 12, pp. 2476-2490, Dec. 2004.

[21] S. M. Yu, Z. G. Ma, S. S. Qiu, S. G. Peng, and Q. H. Lin, "Generation and synchronization of $n$-scroll chaotic and hyperchaotic attractors in fourth-order systems," Chinese Phys., vol. 13, no. 3, pp. 317-328, Mar. 2004.

[22] M. E. Yalcin, J. A. K. Suykens, and J. Vandewalle, "Experimental confirmation of 3- and 5- scroll attractors from a generalized Chua's circuit," IEEE Trans. Circuits Syst. I, Fundam. Theory Appl., vol. 47, no. 3, pp. 425-429, Mar. 2000.

[23] S. M. Yu, S. S. Qiu, and Q. H. Lin, "New results of study on generating multiple-scroll chaotic attractors," Sci. China Ser. F, vol. 46, no. 2, pp. 104-115, Feb. 2003.

[24] J. Lü and G. Chen, "A new chaotic attractor coined," Int. J. Bifurc. Chaos, vol. 12, no. 3, pp. 659-661, Mar. 2002.
[25] J. Lü, G. Chen, D. Cheng, and S. Celikovsky, "Bridge the gap between the Lorenz system and the Chen system," Int. J. Bifurc. Chaos, vol. 12, no. 12, pp. 2917-2926, Dec. 2002.

[26] J. Lü, S. M. Yu, H. Leung, and G. Chen, "Experimental verification of multi-directional multi-scroll chaotic attractors," IEEE Trans. Circuits Syst. I, Reg. Papers, vol. 53, no. 1, pp. 149-165, Jan. 2006.

[27] S. M. Yu, Q. H. Lin, and S. S. Qiu, "A family of multiple folded torus chaotic attractors," (in Chinese) Acta Phys. Sinica, vol. 53, no. 7, pp. 2084-2088, Jul. 2004.

[28] S. M. Yu, J. Lü, H. Leung, and G. Chen, "Design and implementation of $n$-scroll chaotic attractors from a general jerk circuit," IEEE Trans. Circuits Syst. I, Reg. Papers, vol. 52, no. 7, pp. 1459-1476, Jul. 2005.

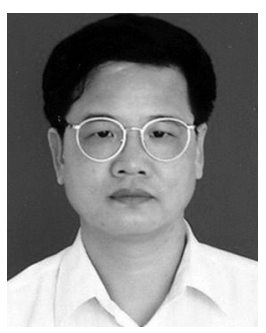

Simin Yu received the B.Sc. degree in electronics from Yunnan University, Kunming, China, in 1983, and the M.E. degree in radio communication engineering and the Ph.D. degree in nonlinear circuits and systems from the South China University of Technology, Guangzhou, China, in 1996 and 2001, respectively.

Currently, he is a Full Professor with the College of Automation, Guangdong University of Technology, Guangzhou, China. His research interests include the design and analysis of nonlinear circuits, computer simulations, and wireless communications.

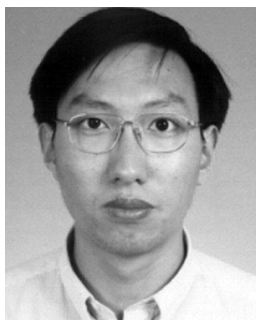

Jinhu Lü (M'03-SM'06) received the Ph.D degree in applied mathematics from the Chinese Academy of Sciences, Beijing, China, in 2002.

Currently, he is an Associate Professor with the Academy of Mathematics and Systems Sciences, Chinese Academy of Sciences, Beijing, China. He held several visiting positions in Australia, Canada, Hong Kong, and USA. From 2005 2006, he was a Visiting Fellow in Princeton University, Princeton, NJ. He served as a member in the Technical Committees of several international conferences and is now a member in the Technical Committees of Nonlinear Circuits and Systems and of Neural Systems and Applications in the IEEE Circuits and Systems Society. He is also an Associate Editor of the Journal of Systems Science and Complexity and a Guest Editor of DCDIS-B. He is the co-author of the most cited SCI paper of Chinese scholars in mathematics in the period of 2001-2005.

Dr. Lü was a recipient of the prestigious Presidential Outstanding Research Award from the Chinese Academy of Sciences in 2002 and the National Best $\mathrm{Ph} . \mathrm{D}$. Theses Award from the Office of Academic Degrees Committee of the State Council and the Ministry of Education of China in 2004.

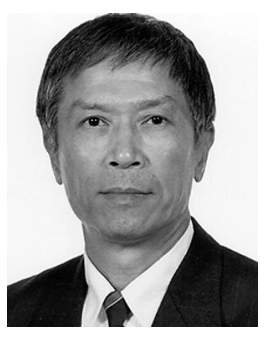

Guanrong Chen (M'87-SM'92-F'97) received the M.Sc. degree in computer science from Sun Yatsen (Zhongshan) University, Guangzhou, China, and the Ph.D. degree in applied mathematics from Texas A\&M University, College Station.

Currently, he is a Chair Professor and the Director of the Centre for Chaos and Complex Networks with the City University of Hong Kong.

Prof. Chen was a recipient of the 1998 HardenSimons Annual Prize for Outstanding Journal Paper from the American Society of Engineering Education, the 2001 IEEE M. Barry Carlton Best Annual Paper Award from the IEEE Aerospace and Electronic Systems Society, and the 2005 IEEE Guillemin-Cauer Best Annual Paper Award from the IEEE Circuits and Systems Society. 